\title{
Atomic carbon in an infrared dark cloud
}

\section{Volker Ossenkopf ${ }^{1,2,3}$, Chris W. Ormel ${ }^{3,4}$, Robert Simon ${ }^{1}$, Kefeng Sun and Jürgen Stutzki ${ }^{1}$}

${ }^{1}$ I. Physikalisches Institut der Universität zu Köln, Zülpicher Straße 77, 50937 Köln, Germany;

${ }^{2}$ SRON Netherlands Institute for Space Research, P.O. Box 800, 9700 AV Groningen,

Netherlands; ${ }^{3}$ Kapteyn Astronomical Institute, University of Groningen, P.O. Box 800, 9700

AV Groningen, Netherlands; ${ }^{4}$ Max-Planck-Institut für Astronomie, Königstuhl 17, 69117

Heidelberg, Germany

Infrared dark clouds (IRDCs) are potential sites of massive star formation, dark in the near-infrared, but in many cases already with indications of active star-formation from far-infrared and submm observations. They are an ideal test bed to study the role of internal and external heating on the structure of the molecular cloud material.

We report the first spectroscopic mapping of an atomic carbon line in an IRDC, G48.650.29 , using the $2 \times 4$ pixel SMART receiver of the KOSMA observatory. Comparing the intensities with the ${ }^{13} \mathrm{CO} 1-0$ data from the BU-FCRAO Galactic Ring Survey (Jackson et al. (2006)) shows a relatively constant line ratio, ranging from about 0.2 to 0.5.

When comparing the influence of the irradiation by embedded sources and by the external Galactic UV field for the production of atomic carbon, we find that most [C I] emission can be attributed to externally illuminated surfaces. A comparable contribution from internal star-formation to the atomic carbon production is only found for the Southern region hosting one of the most evolved embedded sources.

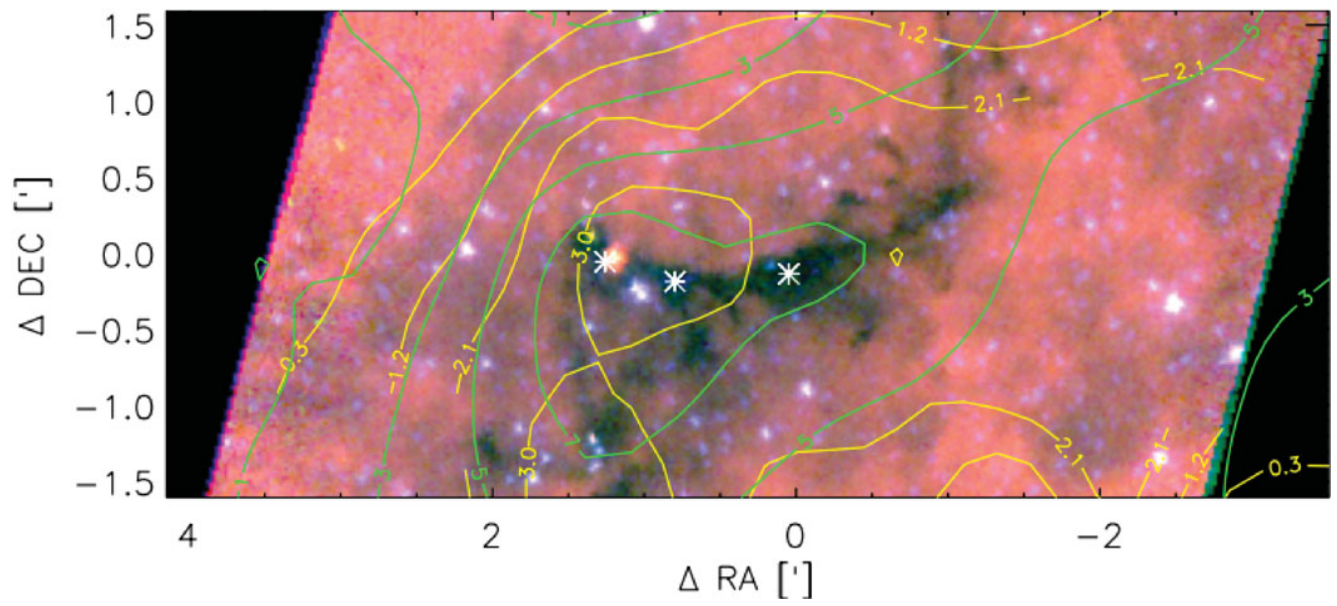

Figure 1. Integrated intensity contours in ${ }^{13} \mathrm{CO} 1-0$ (green) and in $[\mathrm{C} \mathrm{I}]{ }^{3} P_{1}-{ }^{3} P_{0}$ (yellow) on top of a Spitzer IRAC false-colour image, composed of the $8 \mu \mathrm{m}, 5.8 \mu \mathrm{m}$, and $3.6 \mu \mathrm{m}$ channels. The contours are labelled by antenna temperatures integrated from 30 to $41 \mathrm{~km} / \mathrm{s}$. Known submm cores (Ormel et al. (2005)) are indicated by stars.

\section{References}

Ormel, C. W., Shipman, R. F., Ossenkopf, V., \& Helmich, F. P. 2005, A\&A, 439, 613

Jackson, J. M., Rathborne, J. M., Shah, R. Y., Simon, R. et al. 2006, ApJS 163, 145 